\title{
Cultura organizacional y desempeño en instituciones de educación superior: implicaciones en las funciones sustantivas de formación, investigación y extensión *
}

Organizational Culture and Performance in Higher Education Institutions: Implications for the Substantive Functions of Teaching, Research and Outreach

\section{Cultura organizacional e desempenho em} instituições de educação superior: implicações nas funções substantivas de formação, investigação e extensão

\author{
Rosa Angélica González Díaz ${ }^{* \star}$ \\ Sergio Ochoa Jiménez \\ Roberto Celaya Figueroa ${ }^{\star \star \star \star}$
}

Este artículo es un producto central del proyecto de investigación titulado "Estrategia organizacional de restructuración y organización de la profesión académica que responde a las funciones sustantivas de docencia, investigación y extensión en la Universidad de Navojoa".

** Doctoranda en Planeación estratégica para la mejora del desempeño, del Instituto Tecnológico de Sonora (México). Directora de Desarrollo e Innovación Curricular, de la Universidad de Navojoa (México). Correo electrónico: a.gonzalez@unav.edu.mx

*** Doctor en Estudios Organizacionales, de la Universidad Autónoma Metropolitana (México). Profesor de tiempo completo del Departamento de Ciencias Administrativas, del Instituto Tecnológico de Sonora (México). Correo electrónico: sochoa@itson.edu.mx

${ }^{* * * *}$ Doctor en Relaciones Internacionales, de la Universidad de Colima (México), especialista en Consultoría empresarial, liderazgo emprendedor, gestión universitaria y educación superior. Profesor investigador de tiempo completo del Departamento de Contaduría y Finanzas, del Instituto Tecnológico de Sonora (México). Correo electrónico: roberto.celaya@itson.edu.mx 
Fecha de recibido: 13 de enero de 2015

Fecha de aprobado: 24 de julio de 2015

Doi: dx.doi.org/10.12804/rev.univ.empresa.30.2016.01

Para citar este artículo: González Díaz, R. A., Ochoa Jiménez, S. \& Celaya Figueroa, R. (2016). Cultura organizacional y desempeño en instituciones de educación superior: implicaciones en las funciones sustantivas de formación, investigación y extensión. Universidad \& Empresa, 18(30), 13-31. Doi: dx.doi.org/10. 12804/rev. univ.empresa.30.2016.01

\section{RESUMEN}

El terreno de investigación de este trabajo fue una institución de educación superior ubicada en Navojoa, Sonora (México). Se aplicó un modelo de diagnóstico de cultura organizacional que considera siete indicadores. Estos determinan una cultura de innovación en el desempeño de sus funciones sustantivas. Se implementó, en particular, el instrumento adecuado por Sánchez (2010), a partir del trabajo de Cameron y Quinn (1999), con el fin de identificar elementos culturales que limitan o facilitan la implementación de una propuesta de mejoramiento del desempeño. Como resultado, se encuentra que la cultura organizacional no presenta elementos que obstaculizan el proceso, cuando la Alta Dirección asume el compromiso. Se recomienda modificar la propuesta y agregar una etapa inicial de sensibilización.

Palabras clave: cambio organizacional, capacidad de innovación, cultura organizacional, mejora del desempeño.

\section{ABSTRACT}

The fieldwork of this research was carried out in a higher education institution in Navojoa, Sonora (México). Inside it a diagnostic model of organizational culture which refers to seven indicators was applied. These determine a culture of innovation in the performance of its substantive functions. In particular, the instrument adapted by Sánchez (2010) from the work of Cameron and Quinn (1999) was implemented. This was done in order to identify any cultural elements that limit or facilitate the implementation of a proposal for improving the performance. As result, it was found that in organizational culture there are no elements to impede the process when the members of top management team assume the commitment. As a function of the foregoing, it is recommended that the proposal include a sensitization phase at the beginning.

Keywords: Organizational change, innovation capability, organizational culture, performance improvement.

\section{RESUMO}

O terreno de investigação deste trabalho foi uma instituição de educação superior localizada em Navojoa, Sonora (México). Aplicou-se em seu interior um modelo de diagnóstico de cultura organizacional que considera sete indicadores. Estes determinam uma cultura de inovação no desempenho de suas funções substantivas. Implementou-se, em particular, o instrumento adequado por Sánchez (2010), a partir do trabalho de Cameron e Quinn (1999). 
Isto se fez com o fim de identificar elementos culturais que limitam ou facilitam a implementação de uma proposta de melhoramento do desempenho. Como resultado se encontra que a cultura organizacional não apresenta elementos que obstaculizem o processo, quando a alta direção assume o compromisso. Se recomenda, em função do anterior, modificar a proposta agregando ao início uma etapa de sensibilização.

Palavras-chave: mudança organizacional, capacidade de inovação, cultura organizacional, melhora do desempenho.

\section{INTRODUCCIÓN}

Podría ser conveniente comenzar este artículo con la consideración de Celaya (2013):

Los cambios generados desde mediados del siglo XX han desembocado en nuevos marcos conceptuales a través de los cuales el mundo se rige. La globalización y la era de la información son paradigmas principales que actualmente señalan los parámetros y directrices a seguir en un mundo cada vez más interrelacionado. Estos cambios han generado nuevos retos para las profesiones existentes y para los procesos formativos de las mismas (p. 9).

El fenómeno de la gestión educativa, como sucede con muchos otros tipos de organizaciones, debe entenderse desde una visión más amplia, que puede contribuir a movilizar recursos y otros contenidos culturales de una mejor manera, así como a lograr una mayor coherencia entre la cultura y la estrategia. Asimismo, puede aportar a una mejor cohesión de sus miembros en torno al proyecto misional de las instituciones de educación superior.

Para lo anterior, en el contexto de las instituciones de educación superior, es necesario desarrollar estrategias que contribuyan a fortalecer una cultura innovadora, en la que los valores y las conductas predominantes respondan al desarrollo de las funciones sustantivas de formación, investigación y extensión.

Desde esta perspectiva, el presente artículo se concentra en estudiar el importante papel de la cultura en una organización, en particular, en el sector de la educación superior, para su óptimo desempeño. Esto, por medio de la elaboración e implementación de un modelo de diagnóstico cultural que identifique los elementos culturales que favorecen o limitan la puesta en práctica de una estrategia organizacional de restructuración y organización de la profesión académica, en correspondencia con las funciones sustantivas de docencia, investigación y extensión. El terreno de investigación elegido fue 
la Universidad de Navojoa (UNAV) en México.

El texto se ha estructurado en cuatro secciones. En la primera se presenta una revisión de la literatura que considera algunas perspectivas teóricas vinculadas con el tema de la cultura organizacional; en la segunda, la metodología implementada; en la tercera, los principales resultados encontrados gracias a la misma y, en la cuarta, las conclusiones.

\section{REVISIÓN DE LA LITERATURA: PERSPECTIVAS TEÓRICAS DE LA CULTURA ORGANIZACIONAL}

La cultura es un conglomerado social de ideas compartidas, las cuales son matizadas de manera subjetiva por una escala de valores que la colectividad, por sí misma, asigna de una manera particular. El término cultura es muy amplio (Frost, Moore, Louis, Lundberg \& Martin, 1991). Esta, además, puede ser reactiva (dar cuenta de lo que es la sociedad) o proactiva (mostrar lo que puede llegar a ser la sociedad).

Para abordar el estudio de dicho conglomerado, conviene ver a la cultura como un reflejo en el colectivo de creencias, actitudes y valores de los individuos que le dan forma (Schein, 2010) y que, más que como algo definido y definitorio, pueden ser entendidos como un proceso de ser para llegar a ser. Así, cuando se habla de una cultura en tanto reflejo de la colectividad, no es posible dejar de lado cuestiones de tipo filosófico y práctico, en particular, asuntos como la ética y la moral.

La literatura hace referencia a varios tipos de cultura, en función de regiones específicas vinculadas de diversos modos con las organizaciones que se encuentran en ellas (Hofstede, Hofstede \& Minkov, 2010). Se alude, por ejemplo, a culturas como la americana, la europea o la asiática, así como a culturas más específicas como la francesa, la china o la maya. Estas especificidades no son un obstáculo para reconocer que, en la medida en que los seres humanos nos comunicamos e interactuamos, hacemos que la cultura se universalice. Todo parece estar envuelto por un cierto hilo conductor tejido de interrelaciones humanas (Alvear, 2000). Lo anterior plantea la necesidad de que cada pueblo conozca su propia cultura.

La cultura mexicana tiene dos raíces importantes: la tradición prehispánica y la cultura europea, en su variante española. Es un pueblo mestizo con una cultura mestiza (Romero Flores, 1963). De esta manera, cuando se relacionan las tradiciones más importantes de su historia, ambas llegan 
"mutiladas". La cultura mexicana es una sociedad compleja y estratificada en el ámbito rural y urbano y, además, la naturaleza jerárquica que la caracteriza es, tal vez, el principal obstáculo para la implantación de procedimientos de toma de decisiones compartidas y de mejoramiento continuo a largo plazo (Rebolledo, 2003).

Cabe señalar que la forma de solucionar los problemas en la cultura mexicana suele estar sustentada en un liderazgo autocrático, que reduce la participación de los otros y limita la posibilidad de tomar decisiones colectivas y compartidas. Entonces, existe una concepción de la naturaleza humana que promueve la identificación de importantes distancias entre quienes detentan el poder y aquellos que no son poseedores del mismo (Cantú, 2011).

Todo ser humano participa de un modo u otro en la producción de la cultura. Todos somos capaces de poseerla, transmitirla y renovarla. En la cultura no hay copia, pues su construcción siempre implica innovación, lo cual podría conducir a México a una renovación de su cultura para que apalanque una mejora para el país y sus habitantes.

Es posible identificar cuatro tipos de cultura en el contexto empresarial:
1) clan; 2) ad-hoc ("adhocracia"); 3) jerarquizada, y 4) mercado (Cameron \& Quinn, 1999). Con el fin de establecer el nexo entre la cultura organizacional y la efectividad, puede ser relevante generar y conservar una cultura orientada al segundo de estos ("adhocrático"). Este implica que 1) los miembros tienen espíritu emprendedor; 2) los líderes son considerados innovadores y tomadores de riesgo, y 3) la empresa estimula la iniciativa individual (Martínez, Vera \& Serafín, 2014).

De acuerdo con García (2006), la cultura es responsable de los comportamientos. Según Hellriegel y Slocum (2009), si se quiere llevar a cabo un cambio cultural interno en una organización, en función de dichos comportamientos, se deben considerar ciertas pautas básicas en las organizaciones. Estas son: 1) se obtiene lo que se habla; 2) la cultura de trabajo en equipo es reflejo del líder; 3) no es posible ir más rápido que un paso a la vez, y 4) las organizaciones facultadas requieren confianza.

El cambio hacia una cultura que permita la mejora del desempeño debe llevarse a cabo en consideración de la dinámica interna de la organización (Cameron \& Quinn, 1999), de adentro hacia afuera. La tarea es encontrar formas de usar historias, 
leyendas y otros artefactos culturales para mover e influir en los empleados de acuerdo con los propósitos estratégicos (Schein, 1999).

Una práctica habitual en relación con este aspecto, vinculado a la culturadelasorganizaciones, consisteen buscar soluciones para la mejora del desempeño y eliminar, por ejemplo, las que pueden considerarse "manzanas estropeadas". Es usual que cuando este fenómeno se presenta y se trata de eliminar una de esas "manzanas", surja algún otro empleado que también tenga un bajo desempeño, debido a que el comportamiento está determinado por el contexto, entre otros factores y no solo por los aspectos individuales de un sujeto específico. Por esta razón, es necesario detectar, analizar, entender y mejorar el sistema de desempeño en consideración del contexto cultural, lo cual puede traducirse en una mejora durable y sostenida (Bernárdez, 2006).

Para lograr un mejor desempeño en un contexto específico como el de la UNAV, es necesario que sus miembros asuman una actitud proactiva e influyan en el destino de esta institución. Se requiere que todos ellos se apropien de una cultura organizacional que tienda a la innovación para desarrollar una mejora del desempeño a largo plazo. Esto puede generar resultados efectivos y no solo la promulgación de iniciativas promisorias que no se ejecuten en la realidad.

Lo anterior implica ir más allá de declarar lealtad hacia la organización. Consiste en participar de manera constructiva y propositiva en la misma, ser creativos y buscar nuevas experiencias (Weber \& Tina Dacin, 2011). Esto puede hacer que el personal de la institución esté satisfecho con lo que es y lo que hace, lo que, a su vez, puede traducirse en un mayor bienestar (Covey, 2007).

Es preciso que la UNAV sea capaz de gestionar procesos formales que, con el paso del tiempo, se interioricen y se vuelvan parte de la cultura organizacional (Faucheux \& Howell, 1977; Higgins \& McAllaster, 2004). Para esto, es importante proponer mecanismos para valorar, por ejemplo, el impacto de los procesos investigativos en la comunidad tendientes a ofrecer una educación superior coherente y pertinente. Esta responsabilidad impone una reflexión profunda acerca de los propósitos de la educación superior, en cuanto a las formas del ser y hacer docencia, investigación, extensión y vinculación.

Un cambio organizacional, con opcion real de mejorar el desempeño, 
solo será efectivo si se acompaña de un cambio cultural sostenible (Batelaan, 1993; Nyberg \& Mueller, 2009), compuesto por tres fases: penetración, automodificación y autoconsolidación. El valor que otorga el prefijo "auto" es la indicación de que es la propia organización la que ha de determinar su capacidad de cambio (Rodríguez, 1994).

Con base en lo anterior y en los planteamientos identificados en la literatura se hace necesario establecer los elementos culturales básicos que pueden beneficiar u obstaculizar la implementación de la estrategia organizacional y el alcance de un adecuado desempeño. A partir de estas ideas, se presentan algunos postulados que contribuyen a la construcción de un modelo de diagnóstico de cultura organizacional, útil para evaluar lo que sucede en organizaciones como la UNAV. Estos aspectos se sintetizan en la tabla 1 .

Tabla 1. Análisis de modelos de diagnóstico de cultura organizacional seleccionados

\begin{tabular}{|c|c|c|c|}
\hline Autor e investigación & País & Población & Variables consideradas \\
\hline $\begin{array}{l}\text { Hernández (2009) “Cultura } \\
\text { organizacional sólida. Retos } \\
\text { para las IES”. }\end{array}$ & México & $\begin{array}{l}\text { Sección de Estudios } \\
\text { de Posgrado e Inves- } \\
\text { tigación (SEPI) } \\
\text { Instituto Politécnico } \\
\text { Nacional (IPN) }\end{array}$ & $\begin{array}{l}\text { Arquitectura/instalaciones } \\
\text { Herramientas/Tecnología } \\
\text { Obras de arte y literarias } \\
\text { Vestimenta y lenguaje } \\
\text { Ritos, rituales y ceremonias } \\
\text { Formas de comportamiento } \\
\text { Valores, normas y reglas } \\
\text { Creencias y relaciones }\end{array}$ \\
\hline $\begin{array}{l}\text { Martínez y Robles (2009) } \\
\text { "Cultura organizacional } \\
\text { en el sistema de gestión de } \\
\text { calidad en las IES". }\end{array}$ & México & $\begin{array}{l}\text { Dependencias de } \\
\text { educación superior } \\
\text { del Valle de México }\end{array}$ & $\begin{array}{l}\text { Personas } \\
\text { Cliente } \\
\text { Organización } \\
\text { Cultura organizacional }\end{array}$ \\
\hline $\begin{array}{l}\text { Sánchez }(2010) \\
\text { "Caracterización de la } \\
\text { cultura organizacional } \\
\text { de las IES". }\end{array}$ & México & $\begin{array}{l}\text { Instituciones de } \\
\text { educación superior } \\
\text { del estado de } \\
\text { Guanajuato }\end{array}$ & $\begin{array}{l}\text { Características dominantes } \\
\text { Liderazgo organizacional } \\
\text { Administración de los empleados } \\
\text { Cohesión organizacional } \\
\text { Énfasis estratégico } \\
\text { Criterio de éxito }\end{array}$ \\
\hline $\begin{array}{l}\text { Tomas (2010) } \\
\text { "Cultura innovadora } \\
\text { en las universidades". }\end{array}$ & España & $\begin{array}{l}\text { Cuatro universidades } \\
\text { catalanas }\end{array}$ & $\begin{array}{l}\text { Docencia-investigación Universi- } \\
\text { dad-sociedad } \\
\text { Gobierno y gestión } \\
\text { Perfil del profesor } \\
\text { Estudiantado } \\
\text { Evaluación e innovación } \\
\text { Gestión administrativa } \\
\text { Docencia e investigación Univer- } \\
\text { sidad y su contexto }\end{array}$ \\
\hline
\end{tabular}




\begin{tabular}{|l|c|l|l|}
\hline \multicolumn{1}{|c|}{ Autor e investigación } & País & \multicolumn{1}{c|}{ Población } & \multicolumn{1}{c|}{ Variables consideradas } \\
\hline $\begin{array}{l}\text { Vargas (2011) } \\
\text { "Cultura y desarrollo } \\
\text { organizacional". }\end{array}$ & Perú & $\begin{array}{l}\text { Seis facultades de la } \\
\text { Universidad Nacional } \\
\text { del Altiplano }\end{array}$ & $\begin{array}{l}\text { Identidad de los docentes } \\
\text { Compromiso social } \\
\text { Responsabilidad } \\
\text { Profesionalismo } \\
\text { Laboriosidad } \\
\text { Valores }\end{array}$ \\
\hline
\end{tabular}

Fuente: elaboración propia.

Al valorar los modelos presentados en la tabla 1, se evidencia la necesidad de emprender acciones que permitan gestionar una cultura de calidad en las instituciones de educación superior, con el fin de que lo realizado repercuta en el cumplimiento de sus funciones sustantivas.

\section{METODOLOGÍA}

\subsection{Método}

Un constructo complejo como la mejora del desempeño organizacional requiere instrumentos y estrategias variadas que permitan acercarse al objeto de investigación. Por tal razón, este estudio se apoya en una metodología mixta que, de acuerdo con su definición, integra el enfoque cuantitativo y cualitativo (Teddlie $\&$ Tashakkori, 2009). Esto implica considerar dos aproximaciones:

1. Metafórica (cualitativa): los investigadores usan determinados aspectos del lenguaje identificados en documentos, reportes, historias y conversaciones para descubrir patrones culturales.
2. Aproximación cuantitativa: los investigadores utilizan cuestionarios para caracterizar dimensiones particulares de la cultura.

Así, la indagación se enfocó de manera cuantitativa y cualitativa, me diante un trabajo de campo. La investigación es exploratoria (Hernández Sampieri, Fernández Collado \& Baptista Lucio, 2004), ya que se diagnosticó el estado actual de la cultura organizacional en la UNAV y se trató de identificar si esta facilita o no el cumplimiento de sus funciones sustantivas.

El proceso de investigación estuvo compuesto por seis etapas: 1) diseño del modelo de diagnóstico cultural; 2) selección y diseño de instrumentos; 3) aplicación del instrumento seleccionado; 4) procesamiento de la información recabada; 5) análisis de resultados, y 6) generación de conclusiones y recomendaciones.

\subsection{Población}

1. Se trabajó con 32 profesores de tiempo completo de la UNAV, de 
los cuales un $37,5 \%$ pertenece al género femenino y $62,5 \%$ al masculino. Un $9,3 \%$ cuenta con título de licenciatura; un 31,2\% muestra un avance de $50 \%$ en estudios de maestría y un $40,6 \%$ ya tiene el título. Un 3,1\% se encuentra con un avance de $50 \%$ de sus estudios doctorales y un $9,3 \%$ ya cuenta con el grado de doctor.

2. También se trabajó con personal de la Alta Dirección de la UNAV, representado por la Junta de Gobierno. Este órgano está integrado por: rector, vicerrector académico, vicerrector financiero, vicerrector estudiantil, la Dirección de Recursos Humanos, la Dirección de Investigación, la Dirección de Desarrollo Docente, la Dirección de Extensión y Vinculación Universitaria.

\subsection{Instrumentos}

La aproximación cuantitativa comprendió la dimensión organizacional. Esta contempla el modelo de diagnóstico. Refleja los valores fundamentales del desarrollo organizacional y los supuestos implícitos acerca de cómo opera la institución respecto a sus funciones sustantivas. El instrumento trabajado se compone de veinte indicadores que fueron valorados mediante una escala de Likert que va del 1 al 9 .

En este instrumento, cada una de las cuatro áreas que componen la dimensión organizacional (gestión organizacional, liderazgo organizacional, énfasis estratégico y efectividad organizacional) tienen cuatro alternativas, tendientes a caracterizar diferentes enfoques para la UNAV: participación y compromiso, innovación y creatividad, competencia y productividad, y control y eficiencia.

Para utilizar este instrumento era ineludible considerar conceptos, términos e indicadores referentes a una organización educativa, en particular, a una institución de educación superior. Se consideró el Instrumento de valoración de la cultura organizacional (OCAI), adaptado por Sánchez (2010) del trabajo realizado por Cameron y Quinn (1999).

Para probar la confiabilidad y validez del instrumento en el contexto de las instituciones de educación superior, Sánchez (2010) hizo una prueba piloto con veinticinco encuestas. Calculó el alfa de Cronbach para cada cultura y obtuvo los resultados que se exponen en la tabla 2. Estos muestran que el instrumento tiene un adecuado índice de confiabilidad. 
Tabla 2. Medición de confiabilidad del instrumento

\begin{tabular}{|l|c|}
\hline \multicolumn{1}{|c|}{ Cultura } & $\begin{array}{c}\text { Alfa de } \\
\text { Cronbach }\end{array}$ \\
\hline Participación y compromiso & 0,837 \\
\hline Innovación y creatividad & 0,706 \\
\hline Competencia y productividad & 0,873 \\
\hline Control y eficiencia & 0,707 \\
\hline
\end{tabular}

Fuente: Sánchez (2010, p. 178).

Con el propósito de hacer la aproximación metafórica (cualitativa) (ver sección 2.1.), se realizó un análisis de contenido y observación directa para abordar las dos dimensiones restantes propuestas en el modelo: 1) la dimensión núcleo, y 2) la externa. Estas fueron valoradas por la observación y el análisis de documentos institucionales como el Manual de políticas y procedimientos, el Modelo educativo y el Perfil docente.

Para lo anterior, se empleó la técnica que permite estudiar comunicaciones de forma objetiva y sistemática, mediante la organización de los contenidos en categorías. Se usó una escala de calificación del 1 al 5 . Este método permite hacer inferencias válidas y confiables de datos con respecto al contexto. El análisis de contenido siguió la codificación, es decir, la determinación de las características relevantes del contenido en grupos de diez unidades que permiten su análisis detallado por medio de 43 indicadores.

El instrumento se desarrolló con fundamento en los indicadores que señala el Programa Integral de Fortalecimiento Institucional (PIFI), el Programa para el Desarrollo Profesional Docente (Prodep) y la norma ISOO 9001:2008.

Debido a que para datos medidos con una escala de Likert, los porcentajes de cada respuesta pueden resultar más exactos y útiles para sintetizar los resultados (Guerra, 2007), se emplea la escala de Likert que varía del 1 al 5: $1=$ Se realiza con nula frecuencia; 2 Se realiza con poca frecuencia; 3 = Se realiza con mediana frecuencia; $4=$ Se realiza frecuentemente; $5=$ Se realiza siempre.

El detalle de las diez categorías de análisis se muestra a continuación:

Tabla 3. Análisis del instrumento Valoración de la profesionalización docente en relación con el desempeño de las funciones sustantivas

\begin{tabular}{|l|c|c|}
\hline \multicolumn{1}{|c|}{ Área } & Reactivos & Total \\
\hline $\begin{array}{l}\text { Compromiso de la Dirección } \\
\begin{array}{l}\text { Evidencia del compromiso mediante el desarrollo y la imple- } \\
\text { mentación del sistema de gestión de la calidad. }\end{array}\end{array}$ & $1-3$ & 3 \\
\hline
\end{tabular}




\begin{tabular}{|c|c|c|}
\hline Área & Reactivos & Total \\
\hline $\begin{array}{l}\text { Política de la calidad } \\
\text { La Alta Dirección se compromete con la eficacia del sistema } \\
\text { de gestión de la calidad. }\end{array}$ & $4-5$ & 2 \\
\hline $\begin{array}{l}\text { Planeación para la calidad institucional } \\
\text { Actividades orientadas a definir las estrategias y los objetivos } \\
\text { de la Universidad, para promover la mejora de su desempeño } \\
\text { conducente a la calidad institucional y dar respuesta a las } \\
\text { necesidades de la comunidad universitaria y a la sociedad. }\end{array}$ & $6-11$ & 6 \\
\hline $\begin{array}{l}\text { Responsabilidad, autoridad y comunicación } \\
\text { La Alta Dirección se asegura de que las responsabilidades y } \\
\text { autoridades están definidas y son comunicadas dentro de la } \\
\text { organización. }\end{array}$ & $12-13$ & 2 \\
\hline $\begin{array}{l}\text { Revisión por la Dirección } \\
\text { La Alta Dirección revisa el sistema de gestión de calidad a } \\
\text { intervalos planificados para asegurarse de la conveniencia, } \\
\text { adecuación y eficacia. }\end{array}$ & $14-16$ & 3 \\
\hline $\begin{array}{l}\text { Capacitación de cuerpos académicos en el desarrollo de } \\
\text { líneas de investigación de investigación. }\end{array}$ & $17-18$ & 2 \\
\hline $\begin{array}{l}\text { Actualización docente en técnicas y métodos de enseñanza y } \\
\text { en relación con el perfil profesional. }\end{array}$ & $19-20$ & 2 \\
\hline $\begin{array}{l}\text { Vida colegiada: desempeño docente, diseño de proyectos, } \\
\text { seguimiento, evaluación y toma de decisiones. }\end{array}$ & $21-25$ & 5 \\
\hline $\begin{array}{l}\text { Investigación: amplia participación en la investigación de } \\
\text { aporte para la comunidad. }\end{array}$ & $26-36$ & 11 \\
\hline $\begin{array}{l}\text { Vinculación y extensión: amplia participación en las } \\
\text { actividades sustantivas de vinculación y extensión. }\end{array}$ & $37-43$ & 7 \\
\hline
\end{tabular}

Fuente: elaboración propia con base en Programa Integral de Fortalecimiento Institucional (2014), del Programa para el Desarrollo del Profesorado (Prodep) y la Norma ISO 9001:2008.

\section{RESULTADOS}

\section{1. Diseño del modelo}

Tomas (2010), Sánchez (2010), Martínez y Robles (2009), Vargas (2011) y Hernández (2009) han adelantado investigaciones para caracterizar la cultura organizacional en las instituciones de educación superior. Sus aportes se han considerado en este trabajo con el propósito de diseñar un modelo útil para hacer un diagnóstico de la cultura organizacional. El modelo contempla indicadores considerados en el Programa Integral de Fortalecimiento Institucional ([PIFI], 2014) y en el Programa para el Mejoramiento del Profesorado ([Promep], 2008) (figura 1). 
Figura 1. Modelo de diagnóstico cultural (MDC) para la UNAV

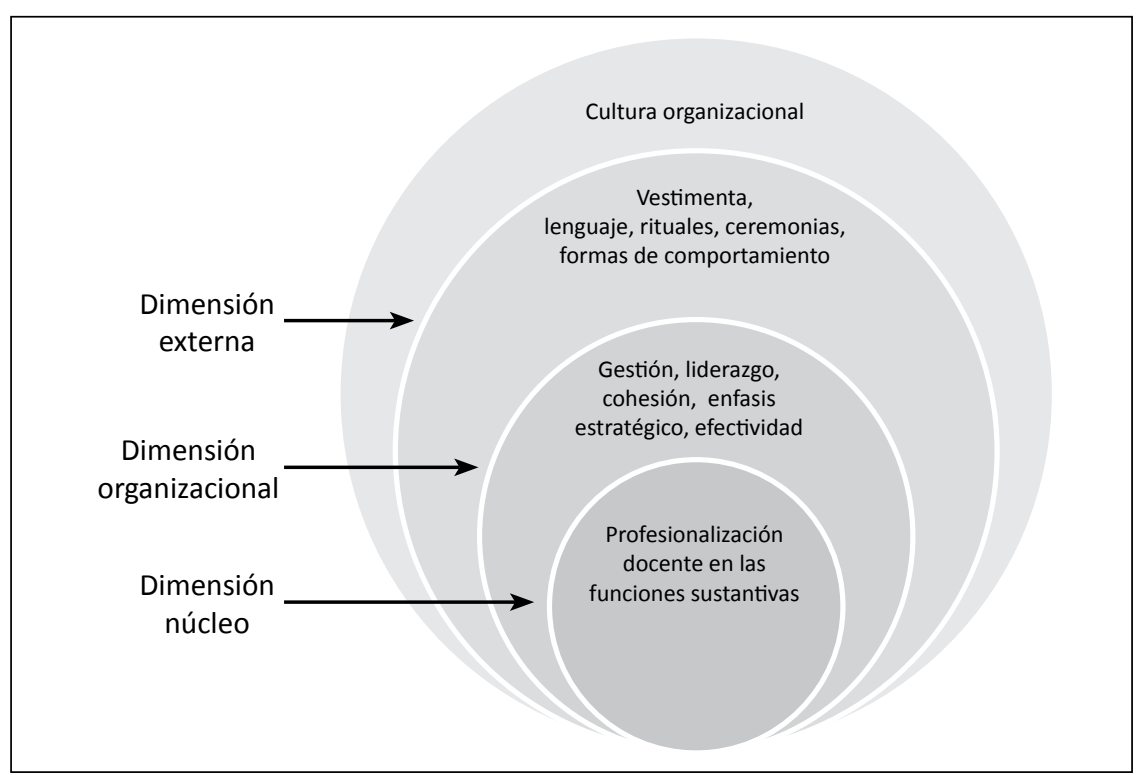

Fuente: elaboración propia con base en Hernández (2009), Martínez y Robles (2009), PIFI (2014), Promep (2008), Sánchez (2010), Tomas (2010) y Vargas (2011).

Como se observa, el modelo de diagnóstico cultural (MDC) propuesto tiene tres dimensiones: 1) núcleo; 2) organizacional, y 3) externa. Estas permiten detectar si el perfil es congruente con las funciones sustantivas de la UNAV en materia de formación, investigación y extensión. Cada dimensión comprende los siguientes elementos:

1. Dimensión núcleo: profesionalización docente en las funciones sustantivas, de formación, investigación y extensión.

2. Dimensión organizacional: gestión organizacional, liderazgo organizacional, cohesión organizacional, énfasis estratégico y efectividad organizacional.

3. Dimensión externa: elementos externos.

\subsection{Aproximación cuantitativa}

La aproximación cuantitativa comprende la dimensión organizacional del modelo. Esta se presenta por medio de un análisis descriptivo en el que se obtuvo una media de 8,9 y representa el valor más alto, lo cual se interpreta como una cultura organizacional orientada al control y la eficiencia (tabla 4). 
Tabla 4. Resultados de cultura organizacional para la UNAV

\begin{tabular}{|l|c|c|c|c|c|c|}
\hline \multicolumn{1}{|c|}{ Cultura } & $\begin{array}{c}\text { Gestión } \\
\text { organizacional }\end{array}$ & Liderazgo & Cohesión & $\begin{array}{c}\text { Énfasis } \\
\text { estratégico }\end{array}$ & $\begin{array}{c}\text { Criterio de } \\
\text { éxito }\end{array}$ & $\begin{array}{c}\text { Promedio } \\
\text { global }\end{array}$ \\
\hline $\begin{array}{l}\text { Participación y } \\
\text { compromiso }\end{array}$ & 1,3 & 2,2 & 1,6 & 2,4 & 1,7 & 1,8 \\
\hline $\begin{array}{l}\text { Innovación y } \\
\text { creatividad }\end{array}$ & 4,3 & 3,1 & 4,8 & 3,6 & 4,2 & 4,0 \\
\hline $\begin{array}{l}\text { Competencia y } \\
\text { productividad }\end{array}$ & 6,2 & 7,4 & 6,7 & 6,1 & 8,2 & 6,9 \\
\hline $\begin{array}{l}\text { Control y } \\
\text { eficiencia }\end{array}$ & 8,6 & 9,2 & 8,4 & 9,3 & 9,1 & 8,9 \\
\hline
\end{tabular}

Fuente: elaboración propia.

En la figura 2 se muestra que coexisten cuatro tipos de cultura, pero predomina la cultura del tipo control y eficiencia, caracterizada por 1) la formalización del trabajo; 2) los procedimientos gobiernan lo que las personas hacen; 3) el estilo de liderazgo es coordinador, monitory organizador; 4) el criterio de efectividad es la eficiencia, y 5) la Alta Administración asume que el control lleva a la eficiencia en la organización.

Figura 2. Resultados de cultura organizacional para la UNAV

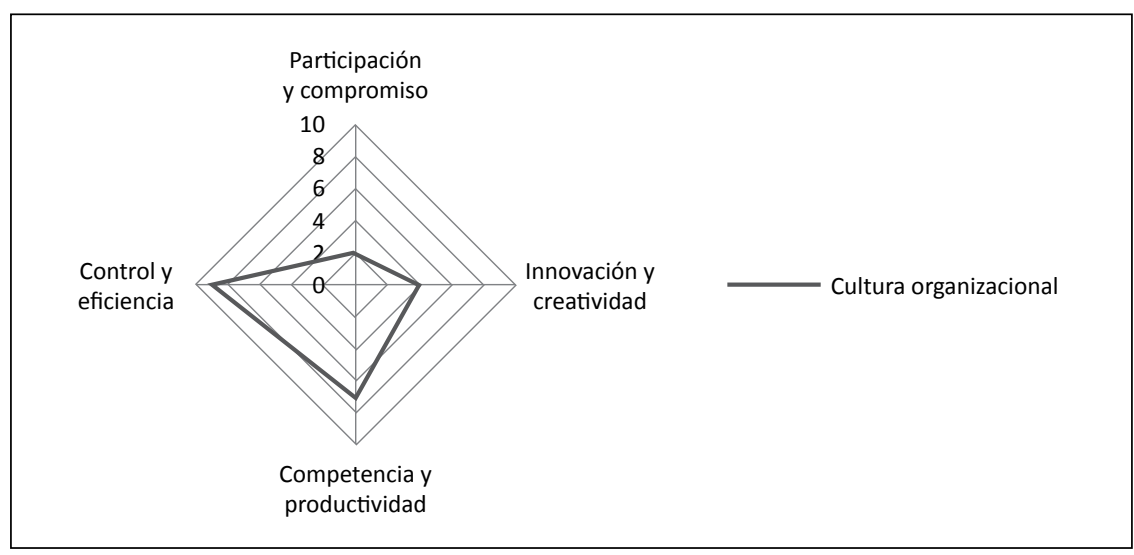

Fuente: elaboración propia.

Los elementos culturales encontra- portamiento distintivo de la organidos con la aplicación del instrumento zación, que orienta a la formalidad, y sus relaciones producen un com- las reglas, los procedimientos de 
operación estándar y una coordinación jerárquica que conduce hacia una cultura burocrática. Tiene como meta la previsibilidad, la eficiencia y la estabilidad. De esta manera, no se observa una fortaleza para la UNAV, ya que el factor que diferencia a las organizaciones de alto desempeño es la congruencia del desempeño organizacional vivido con aquel declarado en sus documentos estratégicos.

En cuanto a las conductas compartidas, se encuentra que un $87 \%$ de los empleados vive en el campus que, en un sentido figurativo, es como una familia; sin embargo, el ambiente de trabajo también se ve influenciado negativamente por esta dinámica, ya que un $67 \%$ de los empleados señala que las recompensas son otorgadas por preferencias y no con base en el desempeño individual.

A su vez, se presenta un sentido fuerte hacia las metas compartidas, ya que un $100 \%$ del personal comparte la filosofía institucional, es decir, los principios característicos de la educación adventista del séptimo día, extraídos de la inspiración bíblica. Estas creencias se ponen de manifiesto en la integración de la fe en la enseñanza. A partir de esta, cada profesor desarrolla una cosmovisión bíblica del curso que imparte, con el fin de que los principios bíblicos y el desarrollo de valores sean trans- mitidos desde cada clase, en el microcurrículo.

Aunque un $92 \%$ de los informantes ha asistido a los cursos de actualización y capacitación en el enfoque de competencias, este no se ve reflejado en el diseño, la implementación y la evaluación de proyectos de impacto en la comunidad. Esto repercute solo en un $0,3 \%$ de productos de investigación generados por año, aun cuando un $92 \%$ de los profesores es de tiempo completo.

La UNAV da cobijo a una planta profesoral híbrida, en la que se yuxtaponen características tradicionales (como el empirismo en su quehacer docente) con características modernizadoras (fundamentadas en una metodología del enfoque por competencias). Existe un predominio de las actividades de docencia fundadas en una tradición de formación de recursos humanos. Esto se refleja en su orientación prioritaria hacia esta función y la relegación de la actividad investigativa, debido a que un $90 \%$ del tiempo de los profesores es dedicado a impartir, planearlas y evaluar clases.

Como resultado de la dinámica que vive la UNAV, el nexo entre investigación y docencia se orienta a brindar un conocimiento codificado de los resultados de investigación 
o a transmitir su experiencia en la aplicación de métodos científicos de investigación. Esto se debe a la usencia de marcos estructurales de orden académico y administrativos que propicien ese vínculo, en particular, por la distribución de carga académica centrada en la actividad de docencia.

Aunado a lo anterior, el profesor acude a impartir clases $y$, después de esto, considera que termina su labor y se va, puesto que no existe una estructura física o académica en donde pueda dedicarse a investigación y extensión. Por esta razón, se alcanzó un $42 \%$ de cumplimiento en el desarrollo de las funciones sustantivas. Así, se observa que la cultura vivida se orienta hacia el control y la eficiencia, mientras la cultura declarada en el modelo educativo y en los fines filosóficos que lo sustentan se enfoca más bien hacia la participación y el compromiso del personal, con una fuerte tendencia hacia la innovación para la producción de conocimiento.

Los símbolos culturales son identificados como un aspecto que fortalece la implementación de la propuesta de mejora, pues se contempla un perfil institucional que instituye aspectos que dan identidad a la organización y que, además, contribuye al proceso formativo del estudiante, porque puede, entre otras cosas, laborar y desarrollar su experiencia en el trabajo manual como una herramienta útil para la edificación del carácter.

\section{CONCLUSIONES}

El mejoramiento continuo, la productividad, la competitividad, la calidad y la innovación con las que se ejecutan las actividades en una organización son elementos detonantes que repercuten en la satisfacción del cliente. Ahí se concentran los esfuerzos de una organización comprometida con la generación de valor a la comunidad en donde se encuentra inmersa y a la que se debe (Guerra, 2007).

Por lo anterior, como generadora del conocimiento, la universidad debe ser gestionada con criterios de eficiencia, efectividad y eficacia, ya que la función de esta institución social no solo consiste en enseñar; en su papel activo — de formación del recurso humano y de gestión de los índices propios de la economía del conocimiento-, también debe aprender a trabajar con los otros actores de la sociedad como la empresa y el Gobierno y articular una triple hélice, en favor del desarrollo de la sociedad (Celaya, 2012; Etzkowitz \& Leydesdorff, 1997).

Para esto, se precisa una cultura organizacional congruente, es decir, 
que el desempeño organizacional vivido esté alineado con el declarado en sus documentos estratégicos. El modelo de diagnóstico cultural que se ha abordado en este trabajo brinda un soporte a la toma de decisiones, por medio de una metodología que permite una amplia y detallada exploración de la UNAV como sistema.

Se contempla la necesidad de incorporar en la propuesta de mejora una fase previa con la Alta Dirección. Esta consistiría en una etapa de sensibilización respecto a las funciones sustantivas propias de una institución de educación superior, en la que los directivos estarían expuestos a dichas funciones, como una demanda primordial de la sociedad. Con ello se pretende diseñar las políticas y la estructura necesaria para que la formación, la investigación y la extensión sean una realidad palpable en la dinámica cotidiana de la UNAV, con el propósito de que la Alta Dirección adopte e impulse una estrategia organizacional que articule las funciones sustantivas, con pleno uso de una gestión y de un liderazgo que tiendan hacia la innovación.

Cabe señalar que la UNAV cuenta con elementos significativos que fortalecen la implementación de la propuesta, entre los que se encuen- tran unos valores organizacionales fortalecidos y compartidos por sus miembros. Estos conducen a un amplio compromiso del personal para la ejecución de las funciones sustantivas. Por lo anterior, se estima que, si los mencionados elementos son tenidos en cuenta, al implementar la propuesta de mejora no habrá mayor resistencia por parte de los empleados, siempre y cuando exista un verdadero compromiso y una participación activa de la Alta Dirección durante la etapa de sensibilización planteada $\mathrm{y}$, más adelante, en la puesta en marcha de las nuevas acciones.

El hecho de encontrar en la investigación que coexisten culturas diferentes en la UNAV, bajo la capa superficial de una cultura organizacional, lleva a los directivos de las instituciones de educación superior a reconocer la necesidad de diseñar estrategias y de construir una visión compartida. En otras palabras, esto los conduce a aportar a la elaboración de una imagen de futuro anclada en metas, valores, objetivos y una misión que, por su legitimidad y consenso, esté en capacidad de unir a la comunidad educativa en torno a una identidad. Con ello, será posible cumplir con altos estándares de calidad las funciones sustantivas de una entidad tan importante para la sociedad y la economía. 


\section{REFERENCIAS}

Alvear, A. C. (2000). Manual de historia de la cultura. México D. F.: Limusa.

Batelaan, V. J. (1993). Organizational culture and strategy: A study of cultural influences on the formulation of strategies, goals, and objectives in two companies. Ámsterdam: Thesis Publishers.

Bernárdez, L. M. (2006). Tecnología del desempeño humano. Bloomington: AuthorHouse.

Cameron, K. S. \& Quinn, R. E. (1999). Diagnosing and changing organizational culture: Based on the competing values framework. Reading: AddisonWesley.

Cantú, D. H. (2011). Desarrollo de una cultura de calidad. México D. F.: McGraw-Hill.

Celaya, F. R. (2012). Papel de la universidad en la triple hélice. Percepción. Recuperado de http://percepcion.com.mx/noticia/25217/papel-de-la-universidad-en-la-triple-helice

Celaya, F. R. (2013). Educación realmente superior. México D. F.: Instituto Tecnológico de Sonora.

Covey, R. S. (2007). Los 7 hábitos de la gente altamente efectiva. México D. F.: Paidós.
Etzkowitz, H. \& Leydesdorff, L. A. (1997). Universities and the global knowledge economy: A triple helix of university-industrygovernment relations. Londres: Pinter.

Faucheux, C. \& Howell, B. (1977). Strategy formulation as a cultural process. international studies of management \& organization, 7(2), 127-138.

Frost, P. J., Moore, L. F., Louis, M. R., Lundberg, C. C. \& Martin, J. (eds.). (1991). Reframing organizational culture. Londres: SAGE.

García, O. (2006). La cultura humana y su interpretación desde la perspectiva de la cultura organizacional. Pensamiento y gestión, 22, 143-154.

Guerra, I. (2007). Evaluación ymejora continua. Conceptos y herramientas para la medición y mejora del desempeño. Bloomington: AuthorHouse.

Hellriegel, D. \& Slocum, J. (2009). Comportamiento organizacional (12aed.).MéxicoD.F.:Thomson. Hernández Sampieri, R., Fernández Collado, C. \& Baptista Lucio, P. (2004). Metodología de la investigación (3a ed.). México D. F.: McGraw-Hill.

Hernández, J. (2009). Una cultura organizacional sólida, uno de los retos para las instituciones de 
educación superior (IES). Conferencia presentada en el Congreso internacional de la universidad: retos y expectativas. México, D.F., Instituto Politécnico Nacional. Recuperado de http://www. repositoriodigital.ipn.mx/bitstream/handle/123456789/4014/ c y r e u - $2009-077 \% 206$. pdf? sequence $=1$

Higgins, J. M. \& McAllaster, C. (2004). If you want strategic change, don't forget to change your cultural artifacts. Journal of Change Management, 4(1), 63-73.

Hofstede, G. H., Hofstede, G. J. \& Minkov, M. (2010). Cultures and organizations. Software of the mind: Intercultural cooperation and its importance for survival ( $3 a$ ed.). Nueva York: McGraw-Hill.

Martínez, E. \& Robles, C. (2009). Cultura organizacional en el sistema de gestión de calidad en las dependencias de educación superior (DES) Valle de México. Gestión y estrategia, 36, 52-64.

Martínez, R., Vera, M. \& Serafin, V. (2014). Cultura organizacional y efectividad en las pequeñas empresas constructoras de Puebla, México. Revista Internacional de Administración \& Finanzas, 7(4), 79-91.

Nyberg, D. \& Mueller, F. (2009). Strategic Cultural Change and Local Discourses: The Impor- tance of Being Different. Scandinavian Journal of Management, 25(2), 146-156.

Programa de Mejoramiento del Profesorado [Promep]. (2008). Promep. Recuperado de http://www. sep.gob.mx/wb/sep1/sep1_Programa_de_Mejoramiento_del Profesorado_PROM\#.V1Clo9gveUk

Programa Integral de Fortalecimiento Institucional [PIFI]. (2014). PIFI. Recuperado de http://pifi. sep.gob.mx/ScPIFI/GuiaPIFI_2014/pdfs/GuiasPDF/Guia PIFI_2014-2015_.pdf

Rebolledo, N. (2003). Antropología y educación intercultural: perspectivas disciplinarias en México. Comunicación y Política, 10(3), 165-188.

Rodríguez, R. (1994). La sistémica, los sistemas blandos y los sistemas de información. Lima: Universidad del Pacífico de Lima.

Romero Flores, J. (1963). Historia de la cultura mexicana. México D. F.: Costa-Amic.

Sánchez, G. M. (2010). Caracterización de la cultura organizacional de las instituciones de educación superior del estado de Guanajuato. (Tesis doctoral inédita). Universidad Autónoma de Querétaro, Santiago de Querétaro, México.

Schein, E. H. (1999). The corporate culture survival guide: Sense and nonsense about culture 
change. San Francisco: JosseyBass.

Schein, E. H. (2010). Organizational culture and leadership (4a ed.). San Francisco: Jossey-Bass. Teddlie, C. \& Tashakkori, A. (2009). Foundations of mixed methods research: Integrating quantitative and qualitative approaches in the social and behavioral sciences. Thousand Oaks: SAGE.

Tomas, M. (2010). La cultura innovadora de las universidades: estudio de casos. Barcelona: Octaedro.

Vargas, R. (2011). Cultura y desarrollo organizacional en la Universidad Nacional del Altiplano - Puno.COMUNI@CCIÓN:Revista de Investigación en Comunicación y Desarrollo, 2(2), 5-16. Weber, K. \& Tina Dacin, M. (2011). The cultural construction of organizational life: Introduction to the special issue. organization science, 22(2), 287-298. 